\title{
Synergistic Effects of Graphene Oxide and Vascular Endothelial Growth Factor Immobilized in Polycaprolactone Nanofiber as a Candidate for Diabetic Wound Healing
}

\author{
Ghader Nuoroozi $^{1(D)}$, Meisam Omidi ${ }^{2,3}$ (D), Masoumeh Rajabibazl ${ }^{3 *}$ (D), Reyhaneh Hoseinpoor ${ }^{4}$ (D)
}

1. Dept. of Clinical Biochemistry, Faculty of Medicine, Shahid Beheshti University of Medical Sciences, Tehran, Iran

2. Protein Research Center, Shahid Beheshti University, Tehran, Iran

3. Dept. of Tissue Engineering and Applied Cell Sciences, School of Advanced Technologies in Medicine, Shahid Beheshti University of Medical Sciences, Tehran, Iran

4. Dept. of Biotechnology, School of Advanced Technologies in Medicine, Shahid Beheshti University of Medical Sciences, Tehran, Iran

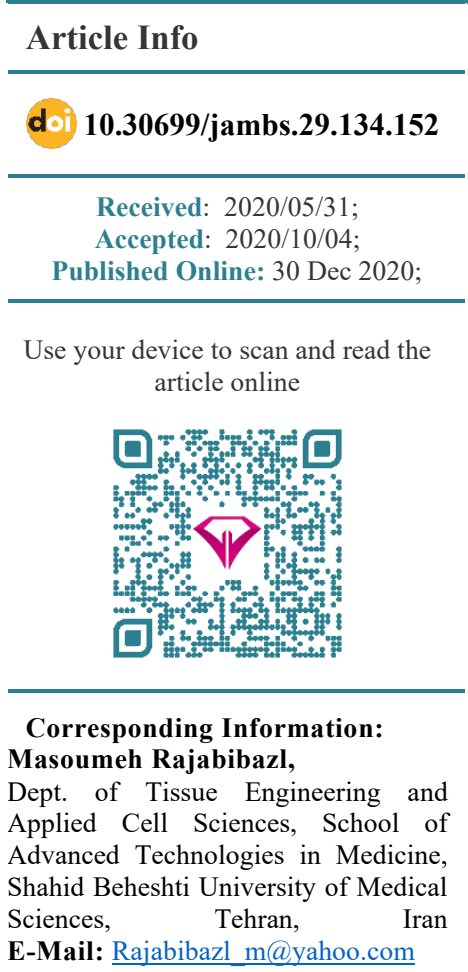

\begin{abstract}
Background \& Objective: The combination of two or more therapeutic agents and their synergetic impacts can be therapeutically effective against multifactorial diseases, such as diabetic foot ulcers. This study demonstrates the application of a nanofiber-based drug delivery system with a controlled release of the growth factor. Various studies have shown that vascular endothelial growth factor (VEGF) stimulates angiogenesis via the VEGF signaling pathway and graphene oxide (GO) has been reported to possess antibacterial property. Therefore, VEGF and GO are hypothesized to have wound-healing effects when used synergistically.
\end{abstract}

Materials \& Methods: In this study, VEGF was purified and verified by western blotting. GO and polycaprolactone (PCL) were prepared by electrospinning and were characterized by scanning electron microscope. Next, VEGF was immobilized by EDC/NHS linker in PCL-GO. Staphylococcus aureus and Escherichia coli were used to evaluate the antibacterial property of GO. Biodegradation and other release properties of the nanofibers were assessed. Moreover, the nanofibers were studied for cell viability and gene expression using human umbilical vein endothelial cells.

Results: The re-analysis of the protein-protein interaction network from the GO database confirmed the centrality of the nitric oxide synthase 3 (eNOS) showing its effects on the expression of other proteins. In addition, the PCL-GO nanofiber loaded with VEGF was studied for the expression of the eNOS gene in the VEGF signaling pathway. It was observed that PCL-GO-VEGF led to an increased expression of the eNOS gene in two weeks.

Conclusion: Based on the observed antibacterial property and angiogenesis influence, PCL-GO-VEGF can be considered as a candidate to promote diabetic wound healing.

Keywords: Graphene oxide, Nanofiber, Nitric oxide synthase type III, Polycaprolactone, VEGF-A

\section{Introduction}

Diabetic injuries are among the chronic wounds currently accounting for a high annual management cost associated with hospitalization, nursing, and subsequent infections (1). These wounds deprive the surrounding tissues of oxygen and nutrients due to ischemia and edema, which fails to restore functional abilities of the skin around the wound site. Therefore, prolonged inflammation and increase in proteases and matrix metallopeptidases (MMPs) occur (2).
Angiogenesis plays a major role in the proliferative stage of wound healing confirmed by the processes of vasodilatation, endothelial cell migration, cell proliferation, blood vessel formation, and a capillary network establishment (3). Vascular endothelial growth factor (VEGF) is one of the potent angiogenic growth factors (GFs) that stimulate multiple phases of angiogenesis-mediated wound healing, such as increasing endothelium permeability and new vessel generation (4). There are different proteins in the 
signaling pattern of VEGF-A/VEGFR2 related to angiogenesis in endothelial cells, some of which play key roles in activating other proteins. Therefore, the usage of bioinformatics databases and reanalysis of genomic data can be beneficial for achieving important hub genes that lead to a better understanding of the mechanisms of such diseases, specifically diabetic ulcer complications (5).

However, due to its short half-life (90 min), VEGF is required in high doses to achieve a therapeutic effect leading to side effects, including the progress of malignant vascular tumors with a higher value of leakage and clearance of GFs from the tissue site (6). Furthermore, the increase of proteases and MMPs at the site of the wound, in response to inflammation in diabetic patients, contributes to the degradation of extracellular matrix and GFs halting the healing process (7). Improvement in drug delivery systems is one of the significant therapeutic requirements for diabetic wound healing. Several advancements have been made in this regard, to mediate the release of active therapeutic compounds. Nanocarriers and biodegradable nanofibers are extensively studied in this aspect, particularly for the controlled release of VEGF, which prevents overdosage $(4,7)$.

Controlled delivery of paracrine factors, such as GFs may significantly elevate tissue remodeling by inducing or inhibiting cell proliferation, survival, and migration. However, the release of GFs from nanofibrous networks has some disadvantages, including protein instability, which is encountered during the hard formulation period and short half-life after release in the tissue. GFs can be loaded into the nanofibrous scaffolds using several strategies, namely bulk encapsulation, surface adsorption, and covalent attachment $(8,9)$.

Synthetic polymeric scaffolds are widely utilized for delivering angiogenic GFs immobilized on their surfaces $(8,10)$. Among aliphatic polyesters with controlled hydrolytic degradation, polycaprolactone (PCL) is an effective option with biocompatibility, low immunogenicity, mechanical properties, and supporting tissue engineering (11). The presence of chemical groups on the surface of PCL, such as amine or carboxylic acids, leads to covalent immobilization of the proteins (12).

Silica nanoparticles have also been applied for the biomolecule delivery system. For example, graphene oxide (GO) has a high loading efficiency due to its two accessible surfaces, high solubility in an aqueous solution, and good biocompatibility (13). Moreover, GO nanoparticles could diffuse easily in the polymer matrix because of oxygenated groups on the surfaces of these nanoparticles, resulting in stable dispersion in organic solvents by electrostatic repulsion (14). Carbon nanotubes have been found to provide effective wound healing characteristics by targeting inflammatory and proliferative phases. As reported previously, GO and the reduced GO nanotube exhibit antibacterial activity $(15,16)$.

This study is aimed to immobilize VEGF in a PCLGO nanocomposite for studying the angiogenic effects resulting from the expression of nitric oxide synthase 3 $(e N O S)$ in human umbilical vein endothelial cells (HUVECs) and evaluating the antibacterial property of GO. Therefore, due to their synergistic effects and with appropriate resealing from the PCL nanofiber, they can be a suitable candidate to promote diabetic wound healing.

\section{Materials and Methods}

\section{Expression, Purification, and Confirmation of Recombinant VEGF-A}

The expression of VEGF-A in E. coli BL21 (DE3) was achieved as previously described (17). However, the sequence of native VEGF-A was inserted into pET32a. In summary, following sonication, the protein was purified by nickel chelate chromatography. Recombinant VEGF was then analyzed on sodium dodecyl sulfate-polyacrylamide gel electrophoresis. Purification of VEGF-A was verified by western blotting using His Tag HRP-conjugated antibody.

\section{Preparation of PCL-GO Nanofibers}

GO nanoparticles were synthesized from graphite powder (Sigma-Aldrich) and the shape, size, and exfoliation of the synthesized GO nanosheets were characterized (18). PCL/granular (Mn 80000, Aldrich) (10 wt \%) was dissolved in a solution of chloroform: methanol (1:4) for one day using a magnetic stirrer. PCL/granular (10 wt $\%)$ and GO nanoparticles (1\%) were dissolved in the PCL solution for the electrospinning process.

Afterwards, $10-\mathrm{mL}$ plastic syringes (gauge 18) were filled with these two solutions and the needle tip was fed with polymer solution by a syringe pump at a flow rate of $1 \mathrm{~mL} \cdot \mathrm{h}^{-1}$. A high-voltage power supply with a positive voltage of $18 \mathrm{kV}$ was used and the collector was applied at $14 \mathrm{~cm}$ in front of the needle. Upon the application of a high voltage $(18 \mathrm{kV})$, a Taylor cone was created from the polymer-nanoparticle solution. Nanofibers were collected on round coverslips $14 \mathrm{~mm}$ in diameter, dried overnight, and stored until use. The morphology of PCL-GO fibers was observed under a LEICA scanning electron microscope (SEM).

\section{Preparation of Protein-loaded PCL-GO} Nanofibers

To immobilize and stabilize VEGF-A and horseradish peroxidase, PCL-GO nanofibrous sheets with varying amounts of surface $\mathrm{COO}$ - and $\mathrm{OH}$ were pre-wetted and hydrated with $\mathrm{NaOH}$ (5 M). The carboxylic groups of PCL-GO scaffolds were submerged in an EDC/NHS mixture that contained $0.0131 \mathrm{~g} \mathrm{~L}^{-1} \mathrm{EDC}$ and $0.0081 \mathrm{~L}^{-1} \mathrm{NHS}$ prior to $10 \mathrm{~min}$ of conditioning with phosphate-buffered saline (PBS). Following washing, the samples were transferred into 
the VEGF-A $(70 \mu \mathrm{g} / \mathrm{mL})$ solution and were incubated for $1.5 \mathrm{~h}$ without shaking and $1.5 \mathrm{~h}$ with shaking at $4^{\circ} \mathrm{C}$. When the coupling reaction was over, the samples were washed three times with PBS.

\section{Degradation and Release}

For degradation measurements, the scaffold specimens were preweighed (Wi) and then incubated in PBS (pH: 7.4 ) at $37^{\circ} \mathrm{C}$ and at different time points for 21 days. Using the dried weight of the scaffold sponges (Wd), the degradation percentage was calculated by the following formula:

$$
\text { Degradation } \%=\mathrm{Wi}-\mathrm{Wd} / \mathrm{Wi} \times 100
$$

In order to evaluate the kinetics of the in vitro release of VEGF-A, the release was predicted using the degradation rate and immobilization amount of the horseradish peroxidase protein in PCL-GO nanofibers. In addition, the specific extinction coefficient of peroxidase $(\varepsilon)$ was used to determine the amount of immobilization calculated by the following equation at different concentrations:

$$
\mathrm{A}=\varepsilon \mathrm{Cd}=(\varepsilon \mathrm{d}) \mathrm{C} \text {; in a graph of A vs. C }
$$

Where $\varepsilon d$ is the slope, A refers to Absorbance, C represents the concentration of immobilized protein, and $\mathrm{d}$ is the length of the light path in $\mathrm{cm}$.

\section{Characterization of Antibacterial Properties}

Antibacterial properties of PCL and PCL-GO scaffolds were investigated via the optical density (OD) method. Bacteria strains, including S. aureus 25923 as gram-positive bacteria and E. coli 25922 as gram-negative bacteria were purchased from Pasteur Institute, Iran and were used for the following purpose. The scaffolds were soaked in $70 \%$ ethanol overnight, washed three times with PBS, and added into the wells. Next, $5 \mathrm{~mL}$ of tryptic soy broth (TSB) culture medium and $20 \mu \mathrm{L}$ of suspended culture were added to each tube. The tube was shaken in the incubator and OD was measured at $610 \mathrm{~nm}$ utilizing a UV spectrophotometer every $2 \mathrm{~h}$ for the total time of $12 \mathrm{~h}$ (19).

\section{In vitro Cell Cytotoxicity and Cell Attachment}

The HUVECs were obtained from the Pasteur Institute, Iran. The cells were cultured in complete Dulbecco's Modified Eagle Medium supplemented with $10 \%$ fetal bovine serum and containing $1 \%$ penicillin-streptomycin (Stock 100X). To achieve 80\% confluency, the cells were sub-cultured and incubated in $98 \%$ humidified air with $5 \% \mathrm{CO}_{2}$.

For the in vitro cell proliferation on nanofibrous scaffolds, the samples of both scaffold groups, including PCL and PCL-GO-VEGF (with a 3-mm diameter), were soaked in $70 \%$ ethanol for $4 \mathrm{~h}$. Next, the scaffolds were seeded at 6000 cells per well in a 96well plate. PCL was used as the control sample and the 3-(4,5-dimethylthiazol-2-yl)-2,5-diphenyltetrazolium bromide (MTT) assay was performed after $72 \mathrm{~h}$ following the manufacturer's instructions. After $6 \mathrm{~h}$ of incubation, the OD values were obtained at $570 \mathrm{~nm}$.
To study cell attachment on nanofibrous membranes, the scaffolds were cultured with HUVECs for $48 \mathrm{~h}$. For this approach, PCL-GO samples were sterilized and seeded with the corresponding cells at the density of 50000 cells per well in 24-well plates. To study proliferation, culturing HUVECs separately on the nanofibrous groups for $48 \mathrm{~h}$ was followed by incubating the nuclei of cells in the dark for $2 \mathrm{~h}$ and staining with 4',6-diamidino-2-phenylindole (DAPI). Finally, images of the stained cells on the scaffold samples were taken using a fluorescent microscope.

\section{Bioinformatics analysis}

The profile of GSE43950 mRNA expression was obtained from the Gene Expression Omnibus (GEO) database (https://www.ncbi.nlm.nih.gov/geo/). The quantile normalization and changes in gene expression in the dataset were re-analyzed using geWorkbench (version 2.6.0) (http://wiki.c2b2.columbia.edu/workbench/index.php/ Home).

In the analysis, healthy controls $(n=5)$ were compared to long-standing diabetic subjects with a microvascular disease $(\mathrm{n}=5)$ using Student's t-test. Genes with $\log \mathrm{FC} \pm 0.5$ were considered to be differentially expressed to achieve the overlapped and differentially expressed genes. The diabetic foot ulcer genes from the Comparative Toxicogenomics Database (CTD) (http://ctdbase.org/ ) and Coremine (https://www.coremine.com/) were used to confirm the GEO database in the Venn diagram (http://bioinformatics.psb.ugent.be/webtools/Venn/).

The STRING database (https://string-db.org/) was utilized for retrieving the interactions between nodes. Network topology, correlation genes, and hub nodes in the base of the parameter degree were analyzed by the Gephi software tool (20).

\section{Gene Expression Study}

The RNA was extracted from HUVECs seeded on PCL and PCL-GO-VEGF using the TRIzol solution (Yekta Tajhiz Azma, Iran). To obtain high-quality RNA, DNase treatment was performed by the DNase I kit (Yekta Tajhiz Azma, Iran) and the RNA was quantified applying a nano-spectrophotometer. RNA transcription was completed by a mixture of oligo (dT) primers and RNAs were kept at $-20^{\circ} \mathrm{C}$. Based on the mRNA sequences, the primer designing tool of NCBI was employed to find the specific primers.

SYBR green real-time quantitative polymerase chain reaction (qPCR) was performed using the StepOnePlus real-time PCR System (Applied Biosystems, BE) to amplify the related cDNAs. The presence of nonspecific PCR products was determined by melting curve analysis. The results were analyzed using the StepOne Software version 2.3. The mRNA expression of the eNOS gene was compared with the expression of glyceraldehyde 3-phosphate dehydrogenase $(G A P D H)$ gene as a reference gene according to the $\triangle \Delta \mathrm{Ct}$ method of REST-2009@ software. 
The sequences of $e N O S$ and $G A P D H$ primers were as follow: $G A P D H$ (Refseq: 2597) forward: AGCCTCAAGATCATCAGCAATGC, GAPDH (Refseq: 2597) reverse: AGTGATGGCATGGACTGTGGTC, eNOS (Refseq: 4846) forward: AATCCTGTATGGCTCCGAGACC, and eNOS (Refseq: 4846) reverse: TCGAGGGACACCACGTCATACTC). The data were analyzed using the paired or unpaired Student's ttest or one-way analysis of variance (ANOVA). Pvalues $<0.05$ were considered significant for statistical analysis.

\section{Results}

Morphology, Degradation, and Release of Nanofibrous Scaffolds

Based on our optimized electrospinning parameters, relatively uniform and bead-free nanofibers were obtained for PCL-GO as shown in Figure 1a. The SEM images demonstrated that the electrospun nanofibers had a porous structure with random arrangements. The mean diameter of the PCL-GO nanofibers was $10 \mu \mathrm{m}$.

The cumulative and individual VEGF release kinetics were predicted by the levels of degradation and the amount of protein immobilization in PCL-GO nanofibers. A biodegradability study of PCL-GO during 21 days revealed scaffold degradation during the first and second weeks, which diminished during the third and fourth weeks (Figure 1b). Moreover, for determining the amount of immobilized protein on the nanofiber, $\mathrm{A}=\varepsilon \mathrm{Cd}$ was used. The concentration (C) of immobilized protein was calculated at $53.65 \mathrm{ng} / \mathrm{mL}$. The base of the degradation rate and the attachment of enzymes for estimating the cumulative and individual release of VEGF from PCL-GO scaffolds are shown in Figures $1 \mathrm{c}$ and $1 \mathrm{~d}$. a

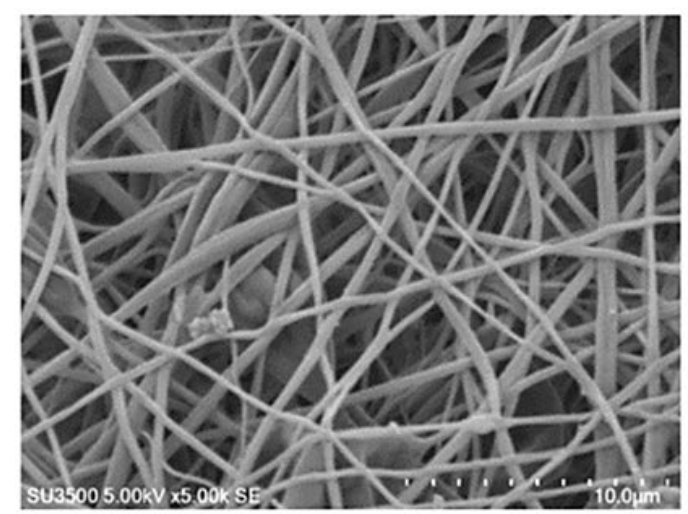

$\mathrm{c}$

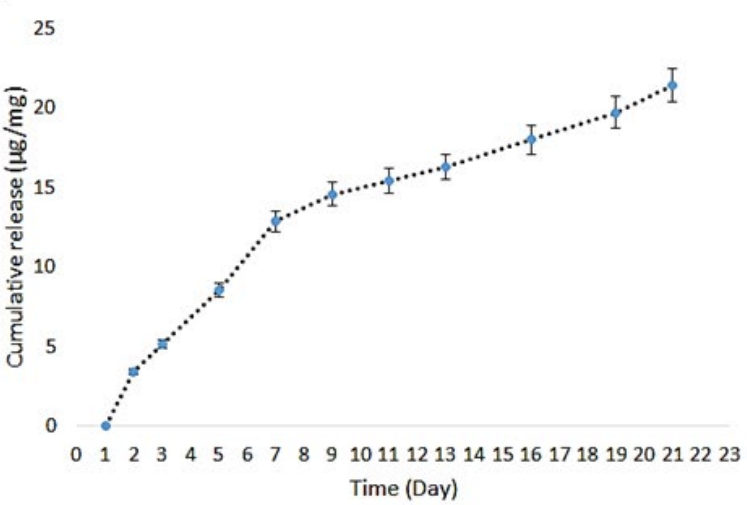

b

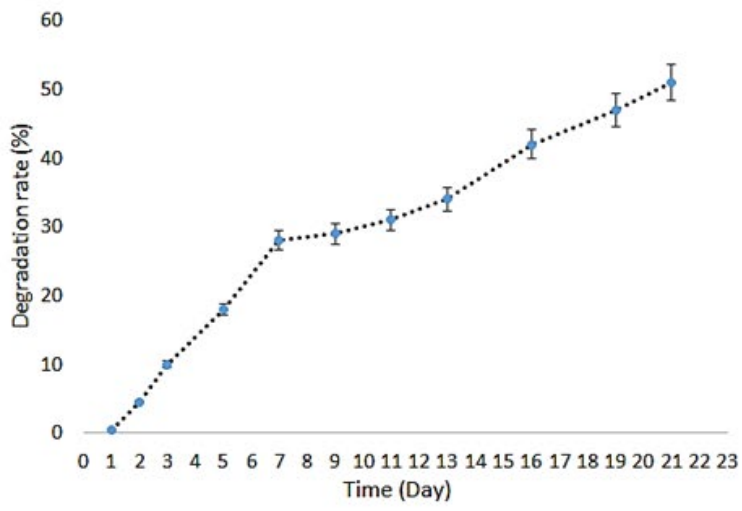

d

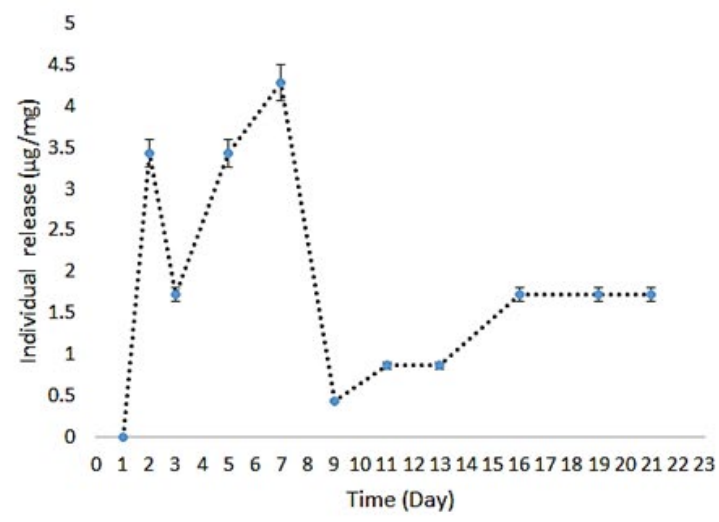

Figure 1. (a) The SEM image of PCL samples containing GO; (b) The degradation rate of PCL-GO; (c, d) estimation of the cumulative and individual release of VEGF $\left(\mathrm{ng} / \mathrm{mL} \mathrm{cm}^{-1}\right)$. 


\section{Antibacterial Properties}

The results of the OD measurements indicated that PCL did not have antibacterial activity, compared to the control (without nanofiber). The growth of $S$. aureus and E. coli in PCL was higher than in PCL-GO. Therefore, PCL-GO nanocomposites inhibit $45 \%$ ( $2 \mathrm{~h}$ ), $35 \%$ ( $12 \mathrm{~h}$ ), and $25 \%$ ( $2 \mathrm{~h}$ and $12 \mathrm{~h}$ ) of the growth of $S$. aureus and E. coli, compared to the control (Figure 2).

\section{In-vitro Cytotoxicity and Cell Attachment}

The distribution pattern of HUVECs on the PCL-GO nanofibers was examined by fluorescence microscopy. The images of nanofibers were taken after $48 \mathrm{~h}$ of cell incubation. The HUVECs seeded on PCL-GO nanofibers showed proliferation, which was confirmed by DAPI staining of the nucleus in blue (Figure 3a). The cytotoxicity of PCL and PCL-GO-VEGF nanofibers was analyzed using the MTT test. Absorbance measurements were used to evaluate cellular proliferation. The results of this test showed that none of the nanofibers inhibited cell growth, in comparison with the control group. On the other hand, PCL-GO was found to have the highest proliferation rate due to the presence of VEGF (Figure 3b).

\section{Protein-protein Interaction (PPI) Network}

The bioinformatic data was re-analyzed and the extracted CTD and Coremine diabetic foot ulcer genes showed that 92 genes were differentially expressed. The results of the Venn diagram demonstrated overlapped differentially expressed genes in the dataset, CTD, and Coremine (Figure 4a). The PPI network of these 92 genes was constructed in the STRING database.

Gephi parameters, such as the degree were applied to assess the topology of the network. This network indicated that eNOS (NOS3) is one of the important hub proteins and VEGF-A is the highest degree hub protein. To discover the pathways that are related to these genes, the KEGG database did not have any overlapping proteins in the VEGF signaling pathway except eNOS (NOS3) (Figure 4b).

\section{Expression of eNOS Gene}

The effects of VEGF on the expression of the eNOS gene in HUVECs and samples treated with PCL and PCL-GO-VEGF were evaluated after 1-2 weeks using q-PCR. As shown in Figure 5, PCL had a basic expression. However, PCL-GO-VEGF led to an upregulation in the expression of eNOS. Moreover, further investigation after 2 weeks revealed an increase in the expression of $e N O S$ in samples treated with PCLGO-VEGF.

\section{S. aureus}

120

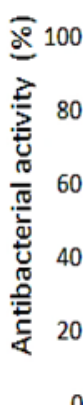

0

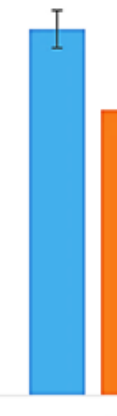

$2(\mathrm{~h})$

$\because$ Contrl $\because \mathrm{PCL} \backsim \mathrm{PCL}-\mathrm{GO}$
E. coli

120

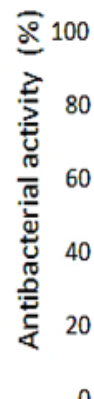

0

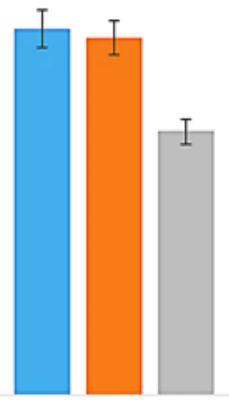

$2(h)$
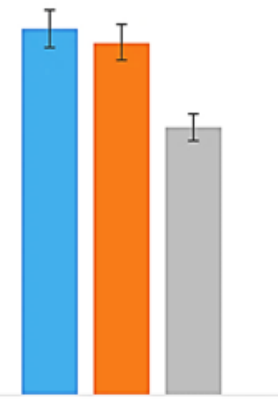

$12(h)$ $\square$ Contrl $\square \mathrm{PCL} \backsim \mathrm{PCL}-\mathrm{GO}$

Figure 2. Optical Density (OD) results of (a) S. aureus and (b) E. coli. with PCL and PCL-GO as compared with the control (without nanofiber) after 2 and12 $\mathrm{h}$. 

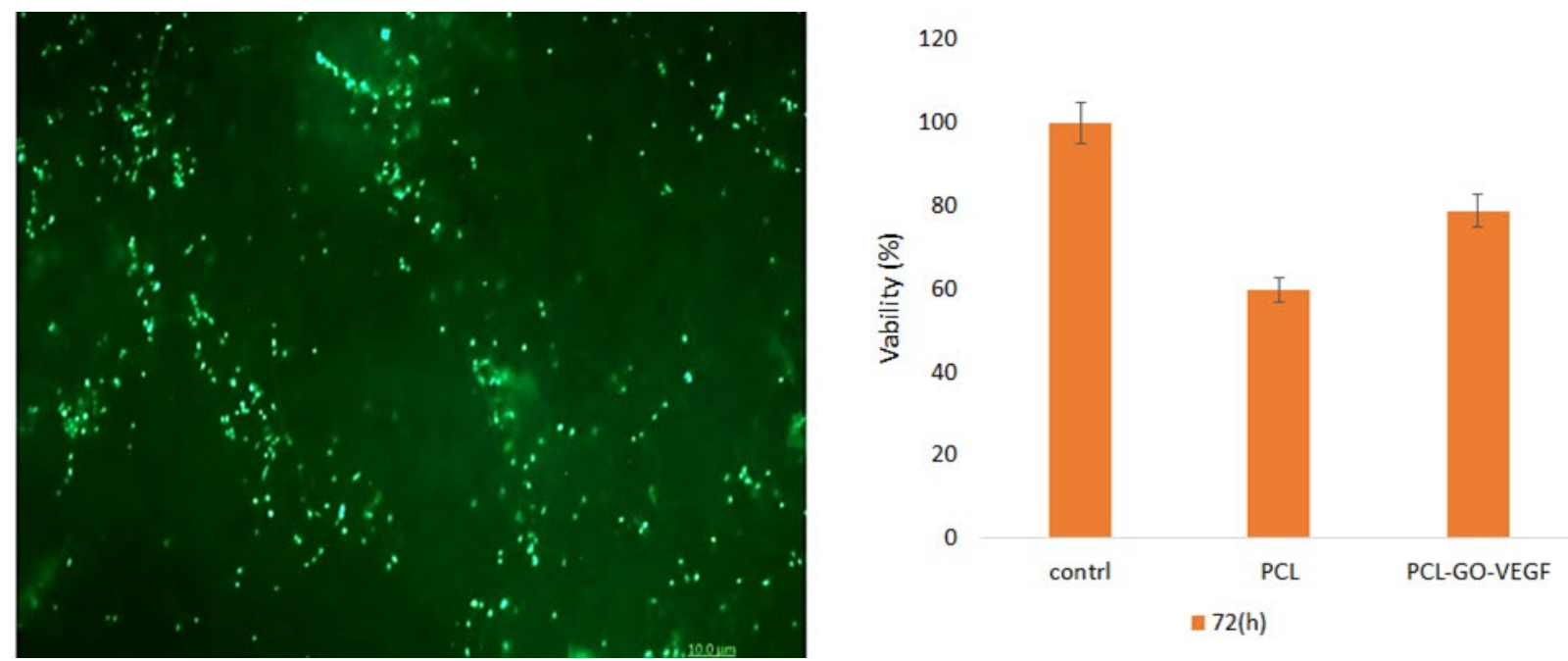

Figure 3. (a) HUVECs attached and spreading on PCL-GO (DAPI staining) (b) Cell viability of PCL and PCL-GO-VEGF nanofiber.

a

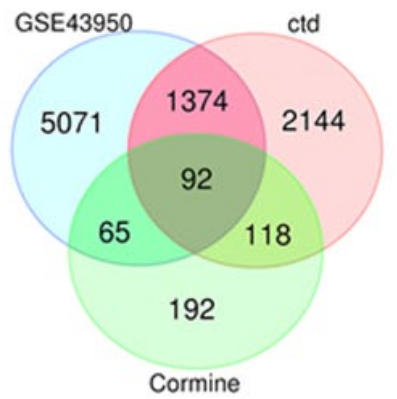

b

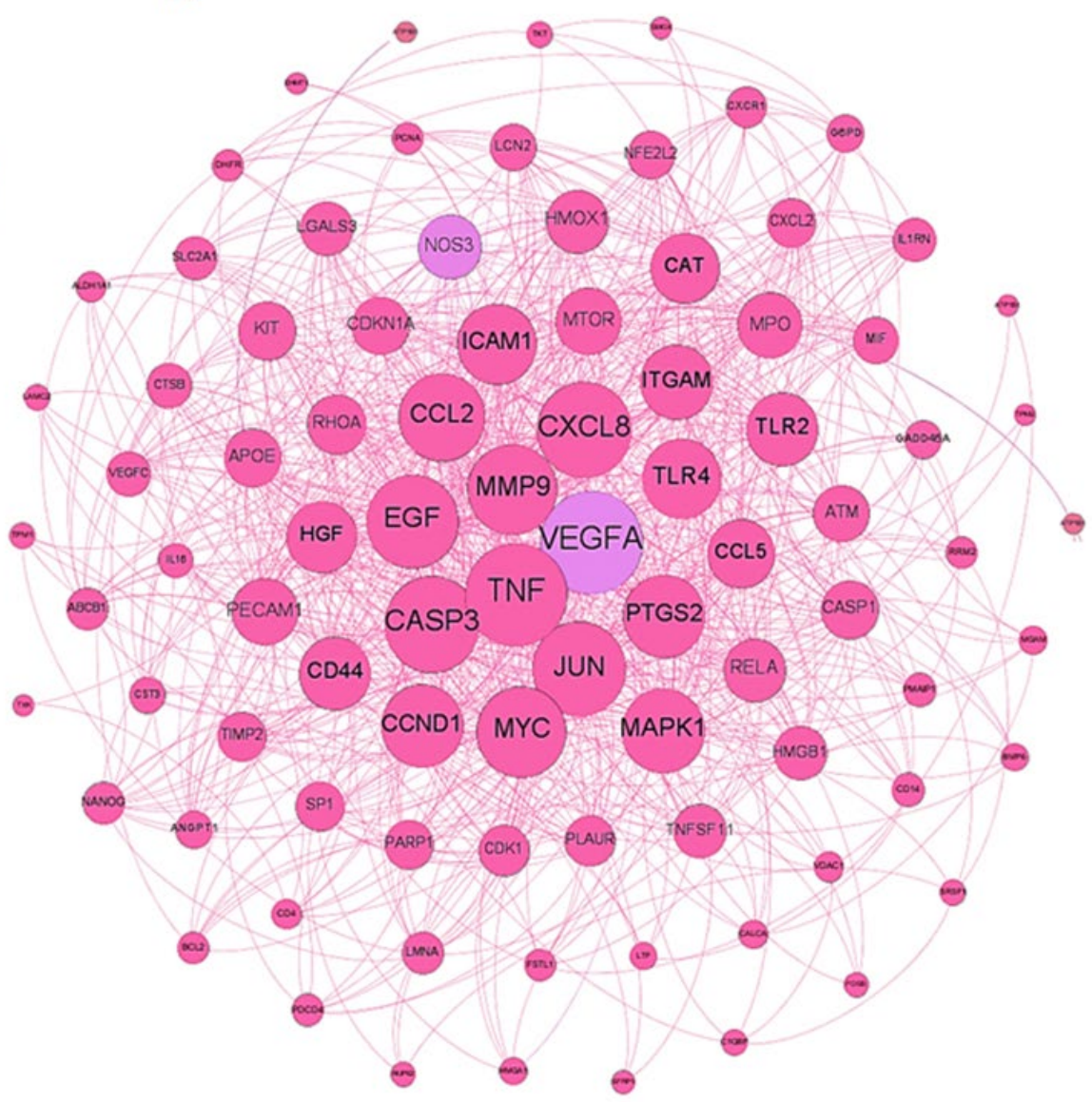

Figure 4. (a) The result of overlapped CTD and Coremine genes with GEO data in Venn diagram. (b) PPI network; in this cluster, NOS3 or eNOS is one of the important hub proteins and VEGF-A is the highest degree hub protein. 


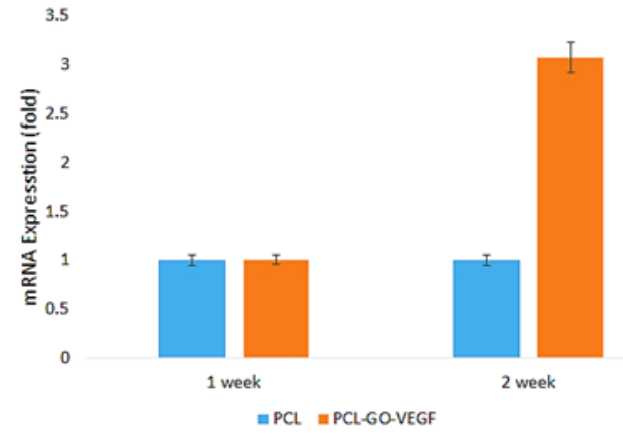

Figure 5. Statistical analysis of eNOS expression in PCLGO-VEGF (weeks 1 and 2) performed by Student's t-test.

\section{Discussion}

Wound healing studies have shown that the inability of diabetic wounds to heal is due to tissue degradation mediated by metalloproteinase and low levels of GFs at the site of the wound. These GFs play a major role in the induction of wound healing (21). Several studies have investigated improved delivery of GFs at the wound site using tissue-engineered scaffolds. Therefore, a drug delivery system that can locally stimulate the controlled release of GFs is required. The present study was designed to construct PCL-based nanofibers with GO and immobilized VEGF to support its controlled release to stimulate diabetic wound healing measured by the expression of $e N O S$.

The GFs commonly need a surface with a hydrophilic nature for covalent immobilization because most GFs can be degraded due to using organic solvents and hence strong covalent bonds are required for the stable and continuous delivery of these factors into cells (22). The enzyme attachment results showed that the attachment of the proteins in the nanofibers was satisfactory and this was confirmed by the bioactivity and stability of the immobilized VEGF.

Degradation due to the absorption of water and enzymatic hydrolysis of intra-polymeric linkages causes VEGF release from the scaffold in the target site (23). In the current study, the in vitro and in vivo release of VEGF from PCL-GO was predicted through estimating protein amount in the scaffold using the bioactivity of horseradish peroxidase protein. In addition, 8 $\mu \mathrm{g} / \mathrm{mg}$ /day VEGF release is required to induce angiogenesis in the tissue (24). Therefore, the VEGF release required for the healing process can be estimated utilizing the biodegradation rate and attached VEGF amount.

Moreover, predictive individual release and cumulative release demonstrated that the amount of VEGF release was appropriate and less than 8 $\mu \mathrm{g} / \mathrm{mg} /$ day. The GO nanostructure exhibited antibacterial activity when gram-positive and gramnegative bacteria were in direct contact with the graphene nanosheets. However, nanocomposites containing GO have not shown significant cytotoxicity in mammals (25).

In order to investigate the antibacterial properties of GO, polyvinyl alcohol-containing chitosan was utilized for a 3\% electro-graphene revealing the antibacterial characteristics of graphene against E. coli. (15). In the present study, PCL containing 1\% GO exhibited antibacterial effects against $S$. aureus and E. coli bacteria.

The eNOS in VEGF signaling pathways has a remarkable role in endothelial cell proliferation and angiogenesis. The reanalysis of the PPI network confirmed eNOS centrality and showed its impacts on the expression of other proteins. In addition, the PPI network analysis demonstrated that VEGF-A can be considered as the highest-degree hub protein. However, the elevation in proteases and MMPs in the inflammatory phase of diabetic foot ulcer led to the degradation of GFs specifically VEGF-A. Therefore, to recognize the effects and mechanisms of VEGF at the molecular level, the expression of the eNOS gene was evaluated using qPCR.

Investigation of the expression of gene samples incubated with HUVECs for 1 and 2 weeks showed that PCL resulted in the basic expression of eNOS. However, eNOS expression augmented in PCL-GO-VEGF and this expression was three-fold higher after 2 weeks. The present study corroborates the existing results that PCLGO-VEGF might be a suitable candidate for promoting wound healing due to its antibacterial and angiogenic influences.

\section{Conclusion}

The current investigation demonstrates the potency of VEGF immobilized in PCL-GO nanofibers for managing chronic wounds. The PCL polymer combined with GO is appropriately porous and nontoxic for growing cells. In addition, it has antibacterial effects against biofilm bacteria in chronic wounds, such as $S$. aureus and E. coli. Reanalysis of the PPI network using genomic databases showed that VEGF and eNOS play a central role in mediating diabetic vasculopathy.

The expression results revealed that VEGF release from PCL-GO nanofibers could lead to eNOS expression for two weeks. Therefore, we can conclude that VEGF might promote the healing process indirectly due to its ability to stimulate angiogenesis and the proliferation of endothelial cells in diabetic ulcers. Finally, because of the antibacterial property and angiogenesis effects, PCL-GO-VEGF can be chosen as a candidate to enhance diabetic wound healing. Further randomized blinded studies can confirm the clinical efficacy and biosafety of these scaffolds. 


\section{Acknowledgments}

This article has been extracted from the thesis written by Mr. ghader Nuoroozi in School of Medicine Shahid Beheshti University of Medical Sciences (Registration No: $467 \mathrm{M}$ with ethical code IR.SBMU.MSP. REC.1395.209 ). The present study was financially supported by fund from School of Advanced Technologies in Medicine from Shahid Beheshti University of Medical Sciences, Tehran, Iran (No: 11450).

\section{Conflict of Interest}

The authors declared no conflict of interest.

\section{References}

1. de Smet GH, Kroese LF, Menon AG, et al. Oxygen therapies and their effects on wound healing. Wound Repair Regenerat. 2017;25(4):591-608. [DOI:10.1111/wrr.12561] [PMID]

2. Tardáguila-García A, García-Morales E, GarcíaAlamino JM, Álvaro-Afonso FJ, MolinesBarroso RJ, Lázaro-Martínez JL. Metalloproteinases in chronic and acute wounds: A systematic review and meta-analysis. Wound Repair Regenerat. 2019;27(4):415-20. [DOI:10.1111/wrr.12717] [PMID]

3. Tahergorabi Z, Khazaei M. Imbalance of angiogenesis in diabetic complications: the mechanisms. Int J Prevent Med. 2012;3(12):827. [DOI:10.4103/20087802.104853] [PMID] [PMCID]

4. Bao P, Kodra A, Tomic-Canic M, Golinko MS, Ehrlich HP, Brem H. The role of vascular endothelial growth factor in wound healing. J Surg Res. 2009;153(2):347-58. [DOI:10.1016/j.jss.2008.04.023] [PMID] [PMCID]

5. Abhinand CS, Raju R, Soumya SJ, Arya PS, Sudhakaran PR. VEGF-A/VEGFR2 signaling network in endothelial cells relevant to angiogenesis. J Cell Communicat Signal. 2016;10(4):347-54. [DOI:10.1007/s12079-0160352-8] [PMID] [PMCID]

6. Geng H, Song H, Qi J, Cui D. Sustained release of VEGF from PLGA nanoparticles embedded thermo-sensitive hydrogel in full-thickness porcine bladder acellular matrix. Nanoscale Res Lett. 2011;6(1):312. [DOI:10.1186/1556-276X-

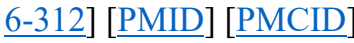

7. Briquez PS, Hubbell JA, Martino MM. Extracellular matrix-inspired growth factor delivery systems for skin wound healing. Adv Wound Care. 2015;4(8):479-89.
[DOI:10.1089/wound.2014.0603] [PMCID]

[PMID]

8. Wang Z, Lu WW, Zhen W, Yang D, Peng S. Novel biomaterial strategies for controlled growth factor delivery for biomedical applications. NPG Asia Materials. 2017;9(10):e435-e. [DOI:10.1038/am.2017.171]

9. Chen RR, Mooney DJ. Polymeric growth factor delivery strategies for tissue engineering. Pharmaceut Res. 2003;20(8):1103-12. [DOI:10.1023/A:1025034925152] [PMID]

10. Praveen G, Sreerekha P, Menon D, Nair SV, Chennazhi KP. Fibrin nanoconstructs: a novel processing method and their use as controlled delivery agents. Nanotechnol. 2012;23(9):095102. [DOI:10.1088/09574484/23/9/095102] [PMID]

11. Brannigan RP, Dove AP. Synthesis, properties and biomedical applications of hydrolytically degradable materials based on aliphatic polyesters and polycarbonates. Biomater Sci. 2017;5(1):9-21. [DOI:10.1039/C6BM00584E] [PMID]

12. Chen S, Li X, Yang Z, et al. A simple one-step modification of various materials for introducing effective multi-functional groups. Colloids and Surfaces B: Biointerfaces. 2014;113:125-33.

[DOI:10.1016/j.colsurfb.2013.08.041] [PMID]

13. Dideikin AT, Vul AY. Graphene oxide and derivatives: the place in graphene family. Front Physics.

2019;6:149.

[DOI:10.3389/fphy.2018.00149]

14. Mahmoudi N, Eslahi N, Mehdipour A, et al. Temporary skin grafts based on hybrid graphene oxide-natural biopolymer nanofibers as effective wound healing substitutes: pre-clinical and pathological studies in animal models. J Mater Sci Mater Med. 2017;28(5):73. [DOI:10.1007/s10856-017-5874-y] [PMID]

15. Lu B, Li T, Zhao H, et al. Graphene-based composite materials beneficial to wound healing. Nanoscale. 2012;4(9):2978-82. [DOI:10.1039/c2nr11958g] [PMID]

16. Khan MS, Abdelhamid HN, Wu H-F. Near infrared (NIR) laser mediated surface activation of graphene oxide nanoflakes for efficient antibacterial, antifungal and wound healing treatment. Colloids and Surfaces B: Biointerfaces. 2015;127:281-91. [DOI:10.1016/j.colsurfb.2014.12.049] [PMID]

17. Ebrahimizadeh W, Gargari SLMM, Javidan Z, Rajabibazl M. Production of novel VHH nanobody inhibiting angiogenesis by targeting binding site of VEGF. App Biochem 
Biotechnol. 2015;176(7):1985-95. [DOI:10.1007/s12010-015-1695-y] [PMID]

18. Afarideh B, Rajabibazl M, Omidi M, Yaghmaee B, Rahimpour A, Khodabakhshi R. Anticancer activity of graphene oxide/5-FU on CT26 DsRed adenocarcinoma cell line. Orient J Chem. 2018;34(4):2002-7. [DOI:10.13005/ojc/3404038]

19. Ul-Islam M, Khattak WA, Ullah MW, Khan S, Park JK. Synthesis of regenerated bacterial cellulose-zinc oxide nanocomposite films for biomedical applications. Cellulose. 2014;21(1):433-47. [DOI:10.1007/s10570-0130109-y]

20. Bastian M, Heymann S, Jacomy M. Gephi: an open source software for exploring and manipulating networks. Third international AAAI conference on weblogs and social media; 2009.

21. Davis FM, Kimball A, Boniakowski A, Gallagher K. Dysfunctional wound healing in diabetic foot ulcers: new crossroads. Curr Diabet Report. 2018;18(1):2. [DOI:10.1007/s11892018-0970-z] [PMID]
22. Masters KS. Covalent growth factor immobilization strategies for tissue repair and regeneration. Macromolec Biosci. 2011;11(9):1149-63. [DOI:10.1002/mabi.201000505] [PMID]

23. Han FY, Thurecht KJ, Whittaker AK, Smith MT. Bioerodable PLGA-based microparticles for producing sustained-release drug formulations and strategies for improving drug loading. Front Pharmacol. 2016;7:185. [DOI:10.3389/fphar.2016.00185] [PMID] [PMCID]

24. Borselli C, Ungaro F, Oliviero O, et al. Bioactivation of collagen matrices through sustained VEGF release from PLGA microspheres. J Biomed Mater Res A. 2010;92(1):94-102. [DOI:10.1002/jbm.a.32332] [PMID]

25. Szunerits S, Boukherroub R. Antibacterial activity of graphene-based materials. J Mater Chem B. 2016;4(43):6892-912. [DOI:10.1039/C6TB01647B] [PMID]

\section{How to Cite This Article:}

Nuoroozi G, Omidi M, Rajabibazl M, Hoseinpoor R. Synergistic Effects of Graphene Oxide and Vascular Endothelial Growth Factor Immobilized in Polycaprolactone Nanofiber as a Candidate for Diabetic Wound Healing. J Adv Med Biomed Res. 2021; 29 (134): 152-160

\section{Download citation:}

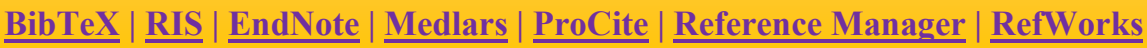

\section{Send citation to:}

Mendeley 2 Zotero (-) RefWorks $\underline{\text { RefWorks }}$ 